\title{
Gamma Ray Bursts from a Quantum Critical Surface
}

\author{
G. Chapline, D.I. Santiago
}

November 20, 2002

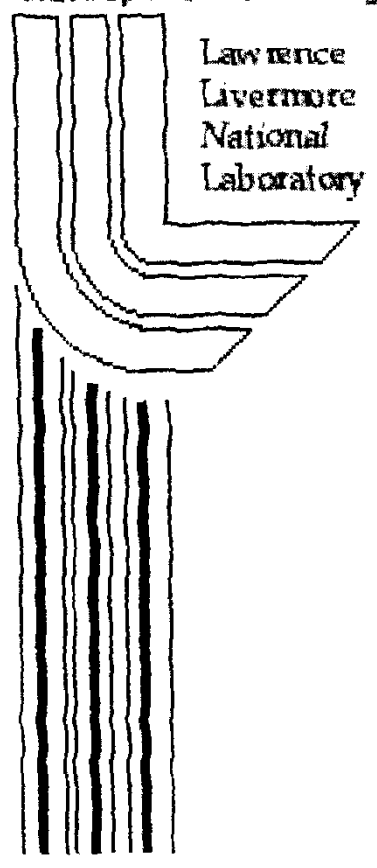




\section{DISCLAIMER}

This document was prepared as an account of work sponsored by an agency of the United States Government. Neither the United States Government nor the University of California nor any of their employees, makes any warranty, express or implied, or assumes any legal liability or responsibility for the accuracy, completeness, or usefulness of any information, apparatus, product, or process disclosed, or represents that its use would not infringe privately owned rights. Reference herein to any specific commercial product, process, or service by trade name, trademark, manufacturer, or otherwise, does not necessarily constitute or imply its endorsement, recommendation, or favoring by the United States Government or the University of California. The views and opinions of authors expressed herein do not necessarily state or reflect those of the United States Government or the University of California, and shall not be used for advertising or product endorsement purposes.

This work was performed under the auspices of the U. S. Department of Energy by the University of California, Lawrence Livermore National Laboratory under Contract No. W-7405-Eng-48.

This report has been reproduced directly from the best available copy.

Available electronically at http://www.doe.gov/bridge

Available for a processing fee to U.S. Department of Energy and its contractors in paper from

U.S. Department of Energy

Office of Scientific and Technical Information

P.O. Box 62

Oak Ridge, TN 37831-0062

Telephone: (865) 576-8401

Facsimile: (865) 576-5728

E-mail: reports@adonis.osti.gov

Available for the sale to the public from

U.S. Department of Commerce

National Technical Information Service

5285 Port Royal Road

Springfield, VA 22161

Telephone: (800) 553-6847

Facsimile: (703) 605-6900

E-mail: orders@ntis.fedworld.gov

Online ordering: http://www.ntis.gov/ordering.htm

OR

Lawrence Livermore National Laboratory

Technical Information Department's Digital Library

http://www.llnl.gov/tid/Library.html 


\title{
Gamma Ray Bursts from a Quantum Critical Surface
}

\author{
George Chapline ${ }^{+}$, David I. Santiago* ${ }^{*}$ \\ + Lawrence Livermore National Laboratory, Livermore, CA 94550 \\ $\dagger$ Department of Physics, Stanford University, Stanford, CA 94305 \\ - Gravity Probe B Relativity Mission, \\ Stanford University, Stanford, CA 94305
}

(November 20, 2002)

\begin{abstract}
The logical inconsistency of quantum mechanics and general relativity can be avoided if the relativity principle fails for length scales smaller than the quantum coherence length for the vacuum state. Ordinarily this corresponds to energies near the Planck energy, but recently it has been pointed out that near to the event horizon of a black hole the coherence length can be much larger and Planck scale physics can take over at macroscopic distances from the event horizon. This has dramatic consequences for the phenomenology of black holes. If we assume that at the Planck scale elementary particles interact via a universal 4-point interaction and baryon number conservation is violated, then the rest mass of a star hitting the event horizon of a large black hole would be rapidly converted into a burst of gamma rays followed by a pulse of hard $\mathrm{X}$-rays whose duration is on the order of the light transit time across the black hole. Predictions for the gamma ray spectra are strikingly similar to those observed for cosmic gamma ray bursts.
\end{abstract}

PACS numbers:

Within the framework of classical general relativity nothing remarkable is expected to happen to an object falling through an event horizon other than the curious circumstance that the observer can no longer communicate with the outside world. Although this prediction has been widely accepted in the physics community, it is inconsistent with quantum mechanics because it conflicts with need for a universal time to define Schrodinger's equation. It has recently been pointed out [1] that this inconsistency can be avoided if it is assumed that as an event horizon is approached, the redshift does not actually go to infinity, but instead spacetime undergoes a continuous quantum phase transition to a de Sitter-like phase. Although we do not have a fundamental theory of this effect, many features of phase transitions, particularly continuous phase transitions, are universal. This universality allows us to make predictions for physical behavior near to the event horizon, and as it happens this predicted behavior is markedly distinct from that predicted by classical general relativity (GR).

As developed previously by us [1], in a simple superfluid model for spacetime, an event horizon would correspond to a surface where the velocity of sound vanishes. One could imagine creating such a surface in the laboratory by supposing that the superfluid is in a vertical column. As a result of the increasing pressure in the fluid as a function of depth it could happen that at a certain depth the speed of sound vanishes. What is noteworthy about this setup is that the behavior of sound waves near to the critical surface is both well defined and up to a certain distance from the critical surface and indistinguishable from the behavior of light outside the event horizon of a Schwarzschild black hole. However, in contrast with the behavior of waves or particles as they cross the event horizon of a classical black hole, the sound waves in our thought experiment would not pass through the critical surface in an uneventful way. There are two effects in particular that will be important for us. First, the frequency of waves that are linearly dispersing with wave number under ordinary circumstances will become a quadratic function of wave number as they approach the critical surface, and secondly above a certain frequency the waves will become unstable as they cross the critical surface.

On the basis of this simple quantum model and assuming universality of behavior at the Planck scale, we predict that when matter hits the event horizon of a large astrophysical black hole it will be affected in a dramatic way. In particular, in the case of a massive black hole like those thought to inhabit the center of many galaxies, we expect that most of the rest mass of a star hitting the black hole event horizon will be converted into a burst of gamma radiation as illustrated in figure 1 .

FIG. 1. Lorentz flattened star approaching event horizon of large black hole. Critical 4-point interactions give rise to nucleon decays into 3 decay products which are visible in the backward direction.

The actual fate of matter hitting the event horizon of a black hole can be surmised from the evolution of the sound waves in our thought experiment as they impinge on the critical surface. It follows from the Schrodinger equation for a superfluid that the dispersion relation for small amplitude waves approaching a critical surface in a superfluid will have the form 


$$
\hbar \omega_{q}=\sqrt{\left(\hbar v_{s} q\right)^{2}+\left(\frac{\hbar^{2} q^{2}}{2 M}\right)^{2}},
$$

where $v_{s}$ is the velocity of sound. In the case of a black hole $v_{g}$ corresponds to $c\left(z / 2 R_{g}\right)$, where $R_{g}=2 G \mathcal{M} / c^{2}$ is the Schwarzschild radius. It follows from equatiom (1)that when relativistic particles with energy $\hbar \omega$ approach to a within a distance

$$
z^{*}=R_{g} \sqrt{\left(\hbar \omega / 2 M c^{2}\right)}
$$

from the event horizon they will begin to behave like non-relativistic particles with mass M. Equation (1) also implies that at sufficiently high energies there will be corrections to the usual special relativistic kinematics, although if $M \sim M_{\text {Planck }}$ these corrections will be much smaller than what has been suggested by others [2].

Although relativistic constituent particles cannot decay, once these particles approach to within a distance $z^{*}$ they can undergo 3-body decays corresponding to the fact that the lowest order interactions at the quantum critical point are 4-point interactions. Since the interactions in the critical region take place at very high energies, one might imagine that the decays shown in Fig 1 are universal for all elementary particles, and can also violate baryon number conservation. Thus diagrams where a quark can decay into an $e^{+}$or $e^{-}$and two antiquarks or a gluon could decay into three $\gamma$ 's would be allowed. The universal rate for these decays would be

$$
\hbar / \tau \simeq(\hbar \omega)^{2} /\left(M c^{2}\right)
$$

Because of the $\omega^{2}$ dependence, the decay rate will be fastest for those constituent particles with the highest intrinsic energies; i.e. the quarks and gluons inside nucleons. Comparing the decay time (3) with the time to cross the critical region of thickness $2 z^{*}$ we find that the decays have time to occur for $\hbar \omega$ higher than about

$$
Q_{0}=10^{9} \sqrt{\left(\frac{M_{\odot}}{\mathcal{M}} \times \frac{M}{M_{\text {Planck }}}\right)} \mathrm{eV},
$$

where $\mathcal{M}$ is the black hole mass.

If the cutoff frequency $Q_{0}$ is below the characteristic energies of the quarks and gluons, i.e. $\sim 100 \mathrm{MeV}$, then the nucleons in a Lorentz flattened star will begin to undergo conversion into $e^{+}$'s, $e^{-}$'s and $\gamma$ rays when the leading edge of the star approaches the critical distance $z^{*}$ from the event horizon surface as shown in figure 1 . We expect that as a result a significant fraction of the entire rest mass of a star will be converted into $\gamma$ rays in a time on the order of the light travel time across the star, i.e. a few seconds, when the star collides with a massive black hole. This results in backward propagating fluorescent radiation with a spectrum of the form

$$
\frac{d N}{d \Omega d \omega^{\prime}}=\frac{27 \mathcal{F}}{16 \pi^{2} \omega} \sqrt{3 \frac{\omega^{\prime}}{\omega}\left[1-3 \frac{\omega^{\prime}}{\omega}-2 \sqrt{\frac{\omega^{\prime}}{\omega}} \cos \theta\right]},
$$

where $\omega$ is the frequency of the incoming constituent particle, $\omega^{\prime}$ is the frequency of the observed radiation, $\theta$ is the viewing angle measured from normal to the event horizon, and $\mathcal{F}$ is the fluence of incoming constituent particles.

Equation (5) predicts that particles radiated backwards from the critical region will be strongly down shifted in energy with respect to the energies of the infalling constituent particles. In the case of nucleons falling onto the critical surface, one can treat the nucleons as collections of noninteracting "partons" [3]. The parton momentum distribution, $p(x)$ as measured in deep inelastic lepton-hadron scattering experiments can be approximately fit [4] by $p_{q}(x)=(1.1+4.1 x)(1-x)^{3.19}$ for quarks and $p_{g}(x)=(2.6+9.2 x)(1-x)^{5.90}$ for gluons. Although the quarks decay immediately into $e^{+}$'s and $e^{- \text {'s }}$, these will eventually be converted into $\gamma$ rays with comparable energies through pair annihilation or synchrotron emmision if there is a strong magnetic field. Therefore the "prompt" $\gamma$ ray spectrum emitted at an angle $\theta$ with respect to the backward direction should be roughly represented by a convolution of the sum of quark and gluon parton distributions with the decay spectrum (5) with a low frequency cutoff $\left(1-e^{-\left(\omega / Q_{0}\right)^{2}}\right)$ :

$$
\frac{d N}{d \omega^{\prime}}=\frac{1}{4 \pi D^{2}} \int \frac{d N}{d \Omega d \omega^{\prime}}\left(\omega, \omega^{\prime}\right) p\left(\frac{\hbar \omega}{m_{\text {proton }} c^{2}}\right) d \omega,
$$

where $p(x)=p_{q}(x)+p_{g}(x)$ and $D$ is the black hole-earth distance. Even though the spectrum (6) depends on angle, because of refraction of trajectories on the way out from the event horizon, the observed spectrum should resemble the $\theta=0$ spectrum for most backward viewing angles. In figure 2 we plot the $\theta=0$ spectrum for $Q_{0}=1 \mathrm{MeV}$, which correspond to $\mathcal{M} \sim 10^{6} M_{\odot}$. For black hole masses greater than $\sim 10^{6} M_{\odot}$ the shape of the prompt spectra will be insensitive to the value of the cutoff $Q_{0}$ and hence we predict that for massive black holes the prompt $\gamma$ ray spectrum will be essentially a universal curve determined by the QCD parton distribution function.

FIG. 2. The backwards fluorescence spectrum for nucleons hitting the critical surface a $10^{6} M_{\odot}$ mass black hole. The solid curve shows a spectrum calculated using an energy distribution for the constituent particles equal to a fit to the nucleon parton distribution function as measured in deep inelastic lepton interaction experiments, while the dotted curve shows an extrapolation of the parton distribution to $Q^{2}=M_{\text {Planck. }}$. The absolute magnitude was fixed by assuming that the source baryon number is that of a $10 M_{\odot}$ star located at a cosmological redshift $\Delta \lambda / \lambda=1.7$. The points show measurements of the prompt gamma ray spectrum from GRB 990123.

Although the "prompt" spectrum shown in Fig 2 was calculated for a single nucleon, the backwards directed $\gamma$ ray spectrum resulting from the initial approach of a 
star approaching the critical surface should look a like the spectrum shown in Fig 3 because the reflected radiation will have such a high energy as viewed in the rest frame of the star that the star will be effectively transparent. Indeed, reversing the momentum of a particle some tens of meters from the event horizon where the red shift would be infinite results in a Lorentz factor $1 / \sqrt{1-v^{2}}$ as seen by the star on the order of $10^{20}$ or larger. The energy loss for such an ultrarelativistic electron, muon, or gamma ray would be negligible.

FIG. 3. Constituent particles with energies less than or on the order of the cutoff $Q_{0}$ can initially pass through the critical surface and then be scattered or decay on their way out as they again cross the critical surface, resulting in an extended pulse whose duration is the light transit time across the black hole.

Constituent particles with energies less than the cutoff frequency (4) will pass through the critical surface (Fig. 3), follow diverging geodesics in the interior of the black hole, and reemerge through the critical surface. Some of this radiation will be redirected into the direction of observation as it passes through the critical surface on the way out. It's a matter of simple geometry that the duration of this extended pulse will be equal to the light transit time across the black hole. In Fig 4 we show a "delayed" spectrum obtained by averaging the decay spectrum (5) over forward angles and cutting off the parton distribution with a factor $e^{-\left(\omega / Q_{0}\right)^{2}}$ with $Q_{0}=1 \mathrm{MeV}$. Because of the refraction of particle trajectories the ratio of time integrated intensities for the prompt and extended pulses will depend on viewing angle. However, in our theory, the spectral shapes of these pulses will be approximately independent of the viewing angle.

These spectra cannot be directly compared with observations of cosmic gamma ray bursts because of the generally unknown cosmological red shifts of the burst sources. However, in a few cases the redshift can be estimated from absorbtion lines in the optical afterglow. For example for GRB 990123 it is known that the redshift $\Delta \lambda / \lambda>1.61$ [5]. Assuming $\Delta \lambda / \lambda=1.7$ we show in Fig 2 how measurements of the initial prompt burst spectrum for GRB 990123 compare with our prompt $\gamma$ ray spectra.

FIG. 4. Extended pulse spectrum corresponding to a $10^{6} M_{\odot}$ mass black hole, obtained by averaging the nucleon decay spectrum, eq 4 , over forward angles.

The approximate agreement between both the absolute intensity and spectral shape of our backward flourescence spectrum and the observed prompt spectrum for GRB 990123 is certainly noteworthy. Perhaps even more remarkably though, the duration and high frequency cutoff of the delayed burst suggest that GRB 990123 resulted from a star hitting $\mathrm{a} \sim 10^{6} M_{\odot}$ black hole. Assuming a cosmological time dilation factor $(1+\Delta \lambda / \lambda)=2.7$, we predict the delayed gamma burst should have lasted about $60 \mathrm{sec}$ and its spectral intensity should be negligible for gamma ray energies above $300 \mathrm{KeV}$, which is consistent with the observations.It should also be noted that the agreement between our prediction for the extended pulse and the GRB 990123 observations can be taken as evidence that the spacetime inside a black hole is just ordinary spacetime with a positive vacuum energy.

To some extent our model resembles the relativistic fireball model [7] for GRBs which invokes a beamed outflow of electrons from an unspecified compact source with a Lorentz factor $>100$. However, in contrast with the fireball model where the gamma rays are generated at distances $>10^{13} \mathrm{~cm}$ from the source via synchrotron radiation, the majority of gamma rays in our model are generated in a region a few light seconds across. The problem of pair creation opacity in a compact source [8] is avoided because our decay leptons and gamma rays are ultrarelativistic with Lorentz factors typically $>10^{10}$ at the critical surface. It should eventually be possible to distinguish our model from the relativistic fireball model because our predictions for how the spectrum and intensity of the prompt and extended gamma ray bursts depend on viewing angle are rather different from what would be expected if the source of the gamma rays was a relativistic beam of electrons.

In summary supefluid model for spacetime proposed in reference 1 predicts that stars hitting the critical surface of a massive black hole will emit $\gamma$ ray pulses with temporal and spectral characteristics strikingly similar to those of cosmic gamma ray bursts. In addition, for black hole masses in the range $10^{6}$ to $10^{9}$ solar masses and cosmological red shifts $1+\Delta \lambda / \lambda$ in the range 2 to 5 , the observed cutoff frequencies in our theory should lie in the range 5 to $500 \mathrm{keV}$. As it happens the photons from cosmic gamma ray bursts typically lie in this range, which perhaps can be considered the first direct evidence that relativity fails for length scales smaller than a quantum coherence length for the ground state of the universe.

\section{ACKNOWLEDGEMENTS}

We would like to thank Robert B. Laughlin for numerous fruitful discussions and suggestions. We also would like to thank Michael Chapline for help with the figures.

[1] Chapline, G., et. al. Phil. Mag. B 81 p. 235 (2001). 
[2] Amelino-Camelia, G., et. al., Tests of Quantum Gravity from Observations of $\gamma$-Ray Bursts Nature 393 p. 763 (1998).

[3] Feynman, R. P., Very High Energy collisions of Hadrons Phys. Rev. Lett. 23, 1415-1417 (1969).

[4] Eichten, E., et. al. Rev. Mod. Phys. 56 p. 579 (1984).

[5] Briggs, M. S. et. al. Astrop. J. 524 p. 82 (1999).

[6] Georgi H. \& Glashow S. L, Unity of all Elementary Particle Fonces. Phys. Rev. Lett. 32, 438-441 (1974).

[7] Goodman Ap J 308, L47 1986, Paczynski Ap J 308, L43 1986.

[8] Piran, T. in "Some Unsolved Problems in Astrophysics" (eds J. N. Bahcall \& J. P. Ostriker) Princeton University Press 1997

This work was performed under the auspices of the U.S. Department of Energy by the University of California, Lawrence Livermore National Laboratory under contract No. W-7405-Eng-48. 


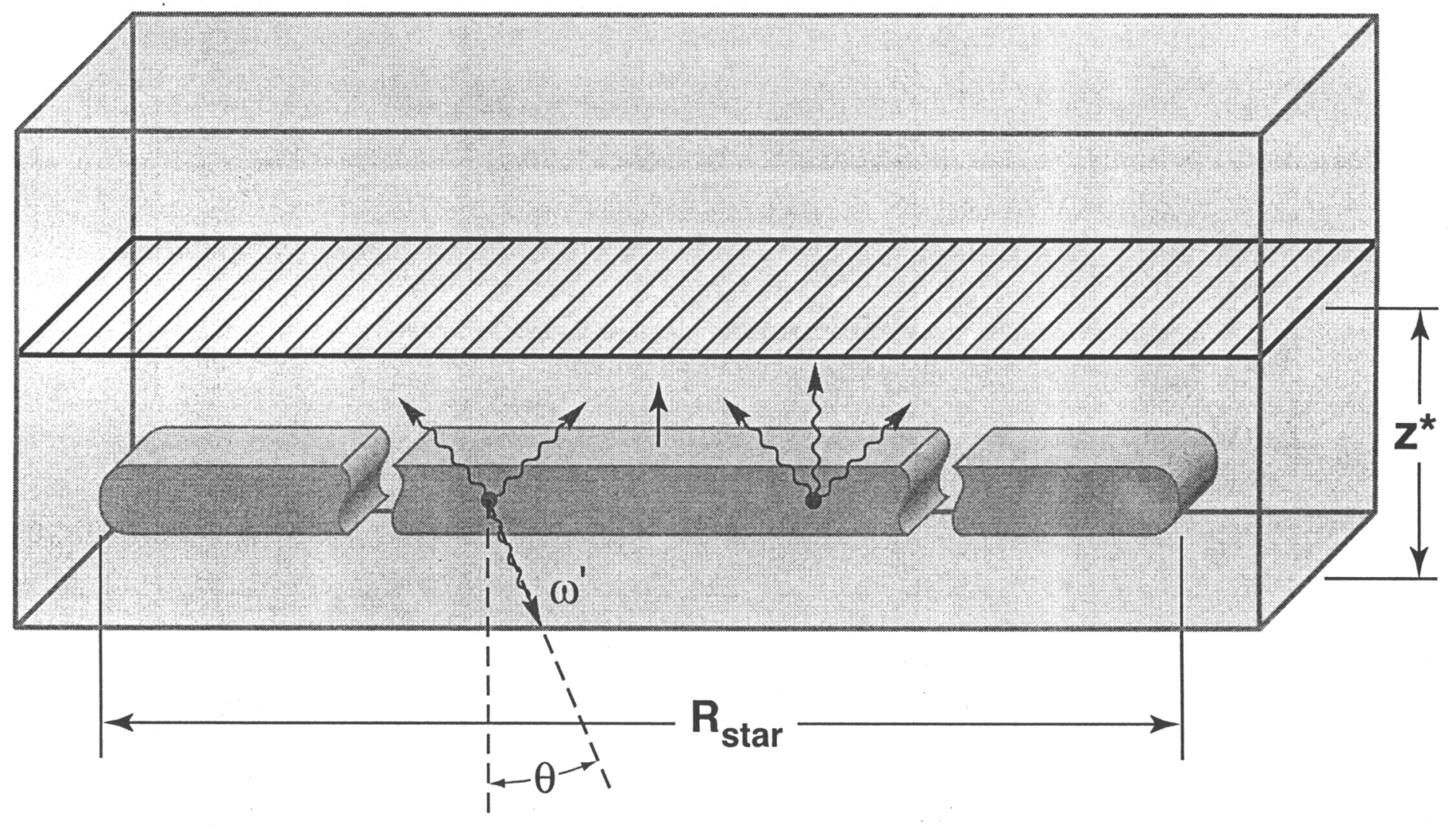




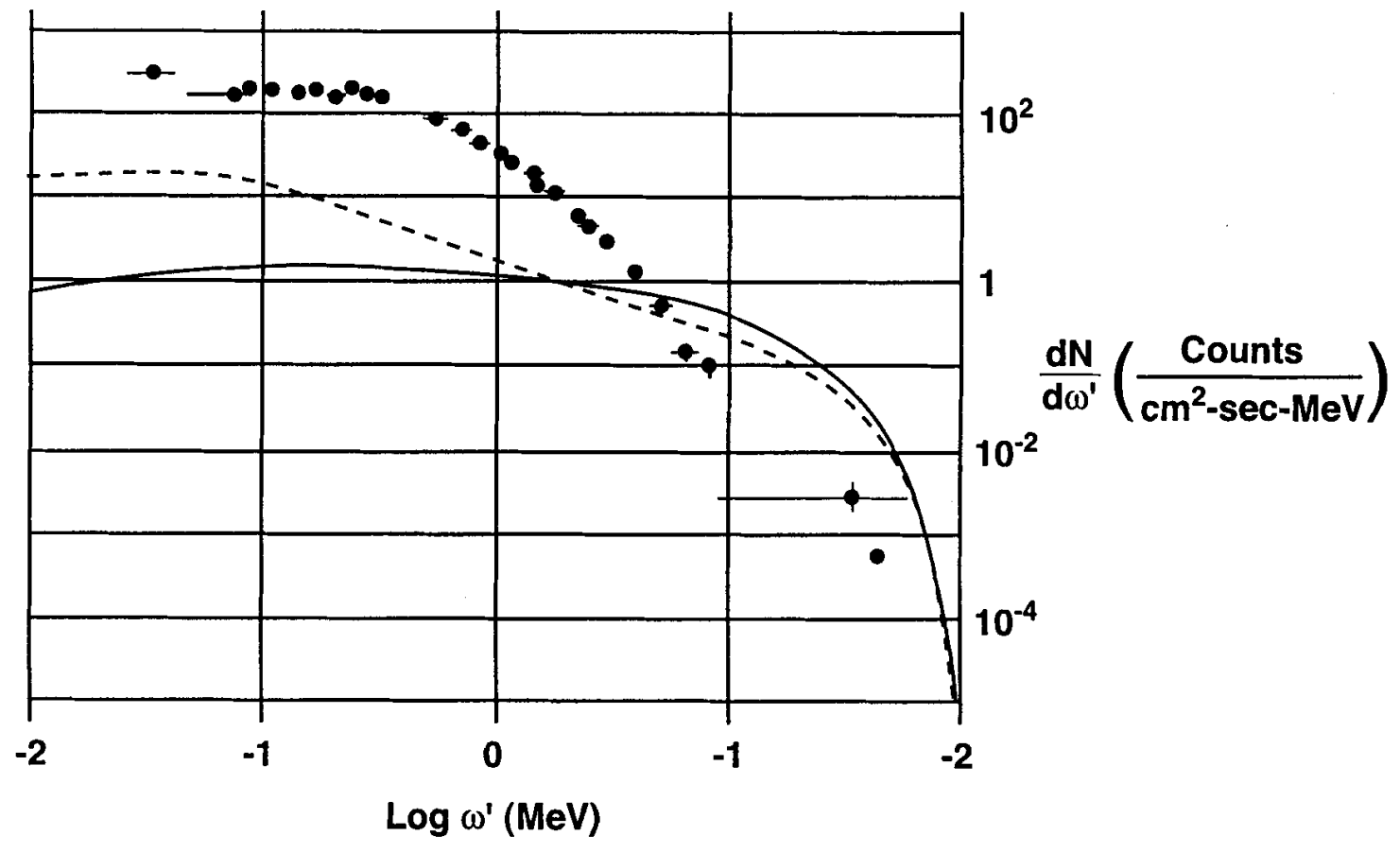




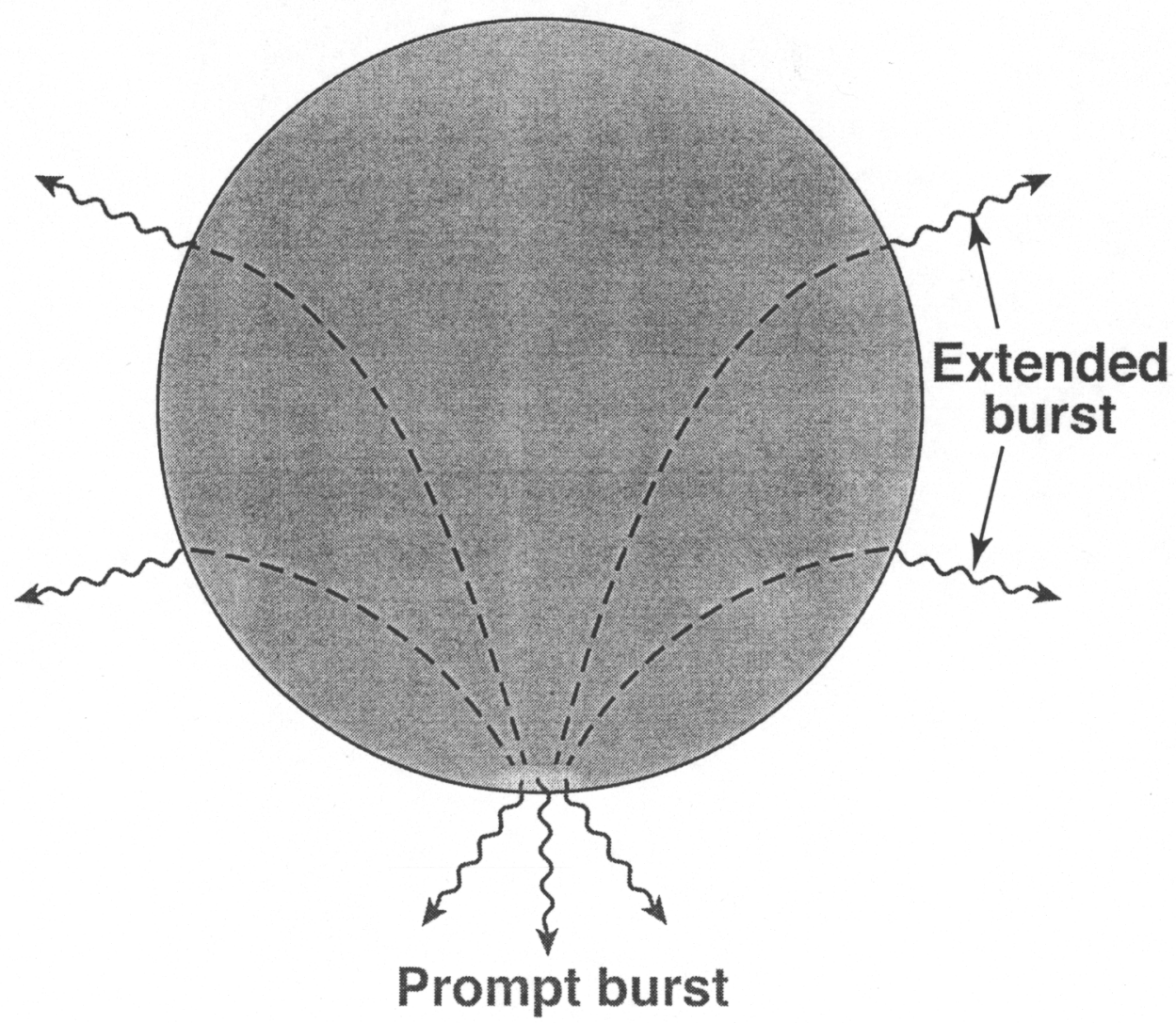




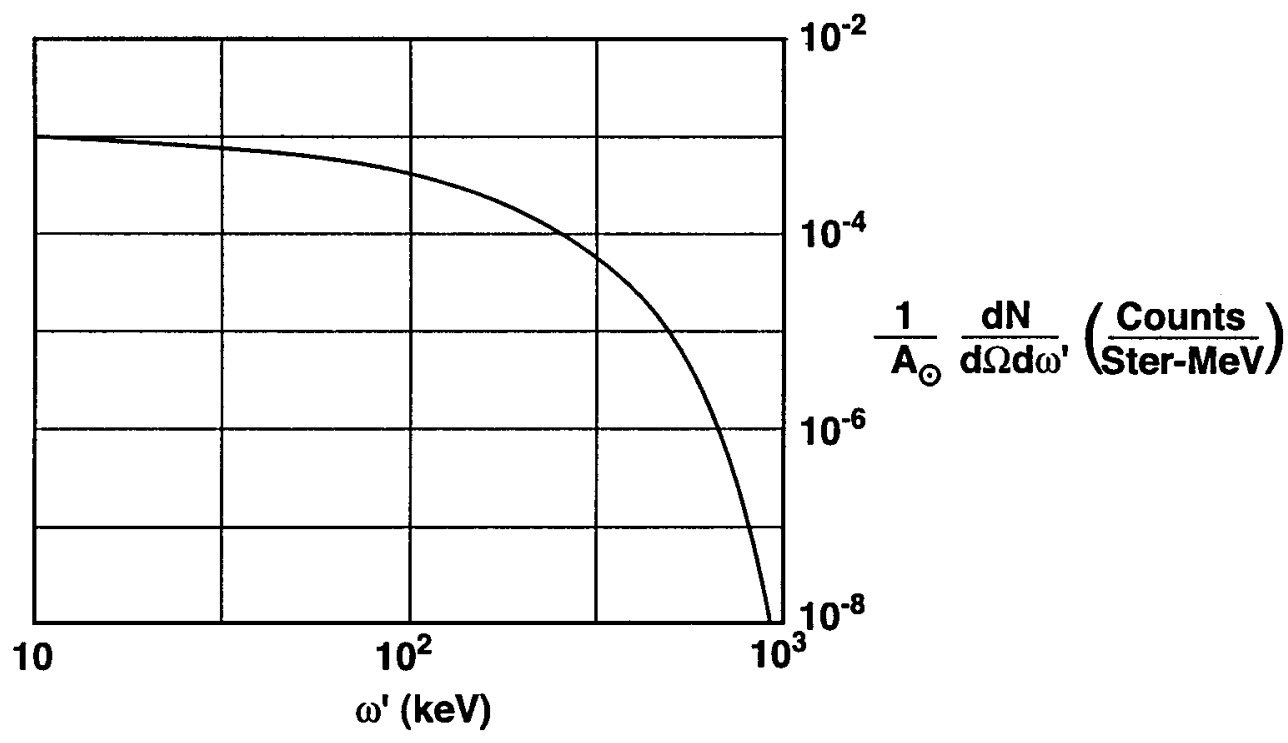

